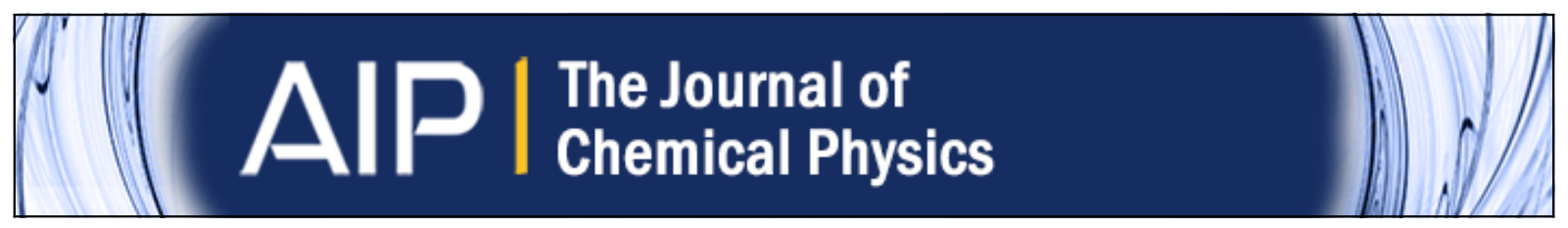

Classical line shapes based on analytical solutions of bimolecular trajectories in collision induced emission. II. Reactive collisions

David Reguera, Philip K. Rawlings, and George Birnbaum

Citation: The Journal of Chemical Physics 138, 224109 (2013); doi: 10.1063/1.4808107

View online: http://dx.doi.org/10.1063/1.4808107

View Table of Contents: http://scitation.aip.org/content/aip/journal/jcp/138/22?ver=pdfcov

Published by the AIP Publishing

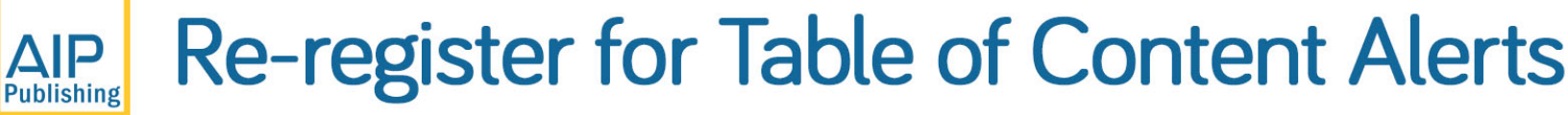




\title{
Classical line shapes based on analytical solutions of bimolecular trajectories in collision induced emission. II. Reactive collisions
}

\author{
David Reguera, ${ }^{1, \text { a) }}$ Philip K. Rawlings, ${ }^{2}$ and George Birnbaum ${ }^{2}$ \\ ${ }^{1}$ Departament de Física Fonamental, Facultat de Física, Universitat de Barcelona, Martí i Franquès 1, \\ Barcelona 08028, Spain \\ ${ }^{2}$ Department of Chemistry and Biochemistry, University of California at Los Angeles, \\ California 90095-1569, USA
}

(Received 8 February 2013; accepted 16 May 2013; published online 13 June 2013)

\begin{abstract}
The classical theory of collision induced emission (CIE) from pairs of dissimilar rare gas atoms was developed in Paper I [D. Reguera and G. Birnbaum, J. Chem. Phys. 125, 184304 (2006)] from a knowledge of the straight line collision trajectory and the assumption that the magnitude of the dipole could be represented by an exponential function of the inter-nuclear distance. This theory is extended here to deal with other functional forms of the induced dipole as revealed by ab initio calculations. Accurate analytical expression for the CIE can be obtained by least square fitting of the ab initio values of the dipole as a function of inter-atomic separation using a sum of exponentials and then proceeding as in Paper I. However, we also show how the multi-exponential fit can be replaced by a simpler fit using only two analytic functions. Our analysis is applied to the polar molecules HF and $\mathrm{HBr}$. Unlike the rare gas atoms considered previously, these atomic pairs form stable bound diatomic molecules. We show that, interestingly, the spectra of these reactive molecules are characterized by the presence of multiple peaks. We also discuss the CIE arising from half collisions in excited electronic states, which in principle could be probed in photo-dissociation experiments. () 2013 AIP Publishing LLC. [http://dx.doi.org/10.1063/1.4808107]
\end{abstract}

\section{INTRODUCTION}

It has been known for a long time that collisions between dissimilar rare gas atoms produce dipoles that absorb and emit radiation from the microwave to the infrared regions. ${ }^{1-3}$ Recently, there has also been interest in collision induced emission (CIE) from very energetic collisions. ${ }^{4-6}$ Here, we consider classical collisions between pairs of atoms that can form a chemical bond and are the type of collisions that are involved in studies of photo-dissociation and chemical reactivity. ${ }^{7}$

The theory dealing with CIA (collision induced absorption) and CIE has been based mostly on a canonical distribution of translational variables and relatively low energy collisions. ${ }^{3}$ By contrast here and in Paper $\mathrm{I}^{1}$ we consider CIE from the viewpoint of analytical solutions of bimolecular trajectories in which energy is conserved. In such a formulation the effect of collisions with a particular kinetic energy can be studied and analytical solutions for the spectrum can be obtained. The spectrum can be calculated using Larmor's classical theory, ${ }^{3,8}$ where the total energy $E(\omega)$ radiated per unit frequency in a single encounter due to an accelerating dipole is given by

$$
\frac{d E(\omega)}{d \omega}=\frac{4 \pi \omega^{4}}{3 c^{3}}|F(\omega)|^{2}=\frac{4 \pi \omega^{4}}{3 c^{3}} I(\omega),
$$

\footnotetext{
a) Author to whom correspondence should be addressed. Electronic mail: dreguera@ub.edu
}

where $\omega=2 \pi c \nu, c$ is the speed of light in vacuum, $v$ is in wavenumber, and

$$
F(\omega)=\int_{-\infty}^{\infty} e^{-i \omega t} \overrightarrow{\boldsymbol{\mu}}(\boldsymbol{r}(t)) d t
$$

is the Fourier transform of the transient dipole of interest, $\overrightarrow{\boldsymbol{\mu}}(\overrightarrow{\boldsymbol{r}}(t))$. Therefore, we can obtain a spectrum if we know: (i) the value of the induced dipole as a function of the interatomic separation $\mu(r)$ and (ii) the separation distance as a function of time $\overrightarrow{\boldsymbol{r}}(t)$, i.e., the trajectory of the colliding atoms.

In Paper I this analysis was restricted to systems in which the induced dipole can be expressed as a single exponential function of the inter-nuclear separation. In the following, we will analyze two different situations. In the first one (Sec. II), we will discuss the CIE arising from half collisions, which is a reasonable model for the dissociation of some excited states of molecules such as $\mathrm{HBr}$. In this case, both the induced dipole and the potential function can be reasonably well represented by a single exponential function. However, such a simple dipole expression does not usually hold for atomic pairs which can form a polar chemical bond. The dipole $\mu(r)$ induced in pairs of dissimilar atoms tends to 0 as $r \rightarrow \infty$ and as $r \rightarrow 0$, thus signifying that there must be at least one maximum somewhere in this range of $r ., 10$ Accordingly, in the second case (Sec. III), we will focus on the CIE due to reactive collisions, where for the induced dipole we will introduce a convenient fit to the ab initio values, and the presence of a well in the interaction will be represented using a Morse potential.

Of the variety of semi-empirical functions that have been proposed to represent the dipole as a function of the 
inter-nuclear separation we choose an exponential representation similar to the form used by Turgov and Chernyavsky ${ }^{11}$ and Ogilvie ${ }^{10}$ We select this form not only for physical arguments justifying it but also because we can make use of the analysis developed in Paper I. ${ }^{1}$ Obtaining an induced dipole function by least squares fitting (least square error (LSE)) a sum of exponentials to $a b$ initio dipole values versus separation distance is described in Sec. III. It will be seen that the spectrum generated by such a dipole differs considerably from the spectrum obtained from a dipole whose variation with inter-nuclear separation can be represented by a single exponential function. The former spectrum (i.e., that due to a peaked dipole function) is more complex and consists of two peaks. The origin of such shapes is discussed in Sec. III, where we also show how a spectrum composed of many terms, originating from the Fourier transforms of the exponential dipole functions, can be well represented by two simple functions whose physical significance may be made apparent. With the aid of this formalism CIE spectra are computed, as an example, for HF. Because of the several approximations involved, these results may have qualitative rather than quantitative significance. However, our purpose here is to obtain formulas that provide reasonable predictions and some guidance in the interpretation of experimental results regarding CIE from the collision of chemically reactive atoms.

\section{CIE FROM HALF COLLISIONS}

Let us start by discussing the CIE spectrum produced by half collisions, an example of which is the case for $\mathrm{H}$ and $\mathrm{Br}$ interacting with a purely repulsive potential function in an excited electronic state. Immediately after $\mathrm{HBr}$ molecules are pumped into such an excited state, the $\mathrm{H}$ and $\mathrm{Br}$ atoms separate with their kinetic energy governed by the repulsive potential to create a half collision. Such collisions may provide a useful way to study experimentally CIE in diatomic molecules thereby avoiding the need to produce two atomic beams. In essence, the study of CIE in such systems blends with that of photo-dissociation.

For the case of $\mathrm{HBr}$ half collisions, both the induced dipole and potential data are known and can each be represented by a single exponential expression. To deal with the above case, we first get the classical trajectory for head-on collisions by solving the kinetic equation for the conservation of energy,

$$
E=\frac{1}{2} m\left(\frac{d r}{d t}\right)^{2}+V(r)
$$

with

$$
V(r)=V_{0} e^{-2 \kappa r},
$$

where $E$ is the fixed total energy, $V_{0}$ is the amplitude, and $2 \kappa$ is the range of the exponential potential energy. The solution for the straight line separation distance versus time,

$$
r(t)=\frac{1}{\kappa} \ln \left(\sqrt{\frac{V_{0}}{E}} \cosh \left(\sqrt{\frac{2 E}{m}} \kappa t\right)\right)
$$

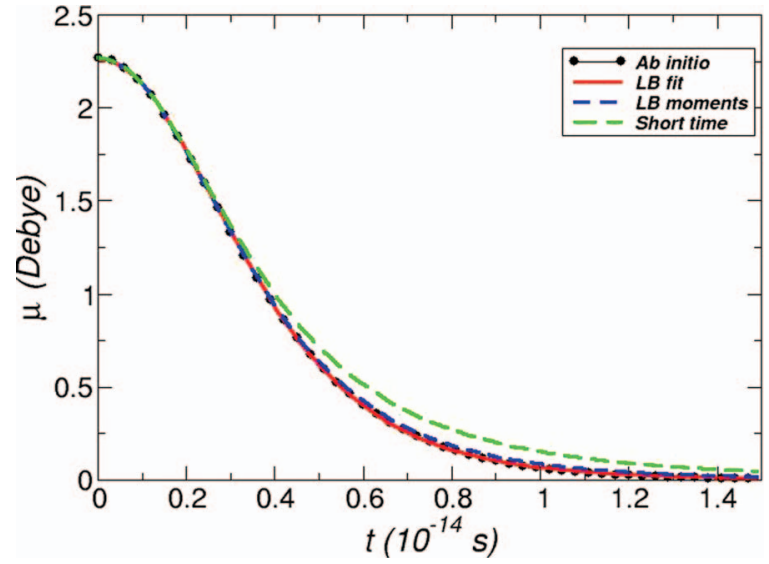

FIG. 1. Dipole versus time for an excited electronic state of $\mathrm{HBr}$ where the potential function is purely repulsive, Eq. (4), and the dipole versus separation distance is exponential, Eq. (6). The points represent the dipole versus time obtained from the kinetic calculations, Eq. (7), and the solid curve represents the best LSE fit of the three LB parameters $C, a$, and $s$ in Eq. (11) to the points. The short dashed curve is computed using the values of the LB parameters obtained from the moment analysis, Eqs. (15) and (16), substituted into Eq. (11). The long dashed curve uses the short time approximation for cosh $(\Omega t)$, Eq. (9).

is then inserted into the dipole exponential function,

$$
\mu(r)=\mu_{0} e^{-\kappa^{\prime} r},
$$

where $\kappa^{\prime}$ is the range of the exponential dipole. The resulting expression for the dipole versus time during a head-on collision is

$$
\mu(r(t))=\mu_{0}\left(\sqrt{\frac{V_{0}}{E}} \cosh [\Omega t]\right)^{-\kappa^{\prime} / \kappa},
$$

where $\Omega=(2 E / m)^{1 / 2} \kappa$. Similar results were obtained by Basile et al. ${ }^{12}$ Unlike previous investigations, we deal here with the case $\kappa \neq \kappa^{\prime}$ which we found to apply for numerous polar diatomic molecules. Figure 1 illustrates the time dependence of the induced dipole given by Eq. (7) for an excited state of $\mathrm{HBr}$, using the parameters listed in the caption of Table I.

In order to get the spectral function $F(\omega)$, the Fourier transform of $\mu(r(t))$ has to be made. By choosing the initial time of the trajectories $t_{0}=0$ at the turning point of the trajectory, the integrand in this case (i.e., for $\mathrm{HBr}$ ) and in the

TABLE I. Comparison of the LB parameters of Eq. (11) evaluated from three different representations of the $\mathrm{HBr}$ excited state spectrum. (a) LSE fit of dipole versus time calculated from Eq. (7). (b) Parameters calculated from the moments of Eq. (7), using Eqs. (15) and (16). (c) Parameters calculated from Eq. (7), using the short time expansion for cosh $(\Omega t)$. For the excited state of $\mathrm{HBr}$, the following kinetic parameter values were used: $\mu_{0}=50 \mathrm{D}$, $V_{0}=1.61 \times 10^{-10} \mathrm{ergs}, E=4.29 \times 10^{-12} \mathrm{ergs}, \kappa=1.22 \times 10^{8} \mathrm{~cm}^{-1}$, and $\kappa^{\prime}=2.08 \times 10^{8} \mathrm{~cm}^{-1}$ estimated from Ref. 17 and reduced mass $m=(80 / 81)$ $1.6725 \times 10^{-24} \mathrm{gr}$.

\begin{tabular}{lccc}
\hline \hline & $C(\mathrm{D})$ & $a\left(10^{-14} \mathrm{~s}^{-1}\right)$ & $s$ \\
\hline (a) LSE fit & 2.2647 & 0.7057 & 3.1823 \\
(b) Moment analysis & 2.2699 & 0.6233 & 2.5582 \\
(c) Short time approx. & 2.2699 & 0.5089 & 1.7054 \\
\hline \hline
\end{tabular}




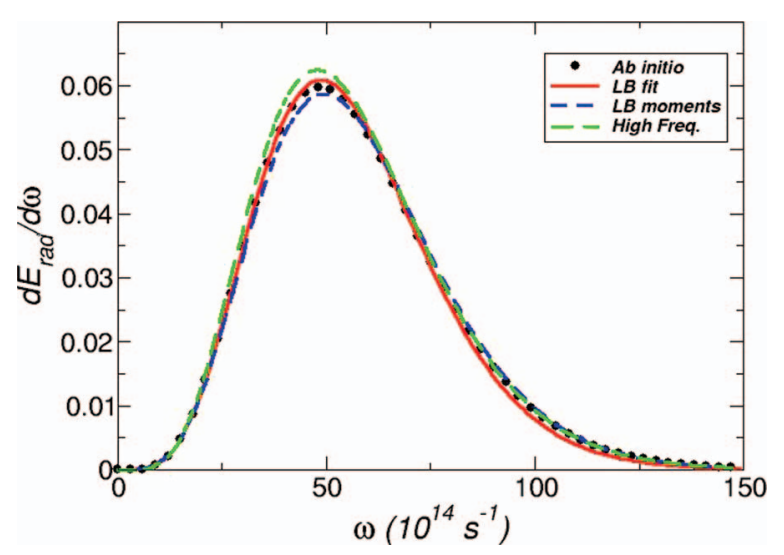

FIG. 2. The spectra, dE/d $\omega$, Eqs. (1) and (2), for an excited electronic state of $\mathrm{HBr}$ are calculated from the curves in Fig. 1. The dots are the spectrum of the kinetic model, Eq. (8). The solid curve is the best LSE fit of the three LB parameters, Eq. (11). The short dashed curve is computed with the LB parameters obtained from the moment analysis, Eqs. (15) and (16). The long dashed curve is calculated from the short time approximation, Eq. (9). In addition, the curve with the highest amplitude is calculated from the high frequency approximation, Eq. (10).

following (i.e., for HF) becomes an even function of time. ${ }^{1}$ Accordingly, we will compute only the cosine transform here and also in the rest of the paper.

The cosine transform of Eq. (7) is (Ref. 13, p. 30, No. 5),

$F(\omega)=\mu_{0}\left(\frac{V_{0}}{E}\right)^{-x / 2} \frac{2^{x-2}}{\Omega \Gamma(x)} \Gamma\left(\frac{x}{2}+i \frac{\omega}{2 \Omega}\right) \Gamma\left(\frac{x}{2}-i \frac{\omega}{2 \Omega}\right)$,

where $x=\kappa^{\prime} / \kappa$. The resulting spectrum, plotted for the excited state of $\mathrm{HBr}$ in Fig. 2, is characterized by the presence of a single peak, which is similar to that obtained previously ${ }^{1,7}$ for collisions between rare gas atoms. The inverse of the quantity $\Omega$ is the approximate duration of collision and controls the width of the spectral function.

A simplification may be obtained using a short time approximation for Eq. (7). At very short times the expansion for $\cosh (\Omega t) \approx 1+0.5(\Omega t)^{2}$ should be reasonably accurate since much of the CIE interaction occurs near the distance of closest approach where $t$ is very small. Substituting this approximation into Eq. (7) gives the following relationship:

$$
\mu(r(t))=\mu_{0}\left(\frac{V_{0}}{E}\right)^{-x / 2}\left[1+\frac{(\Omega t)^{2}}{2}\right]^{-x},
$$

which is plotted for the excited state of $\mathrm{HBr}$ by the longdashed line in Fig. 1.

Alternatively, the high frequency approximation of Eq. (8) is obtained from ${ }^{14}$

$$
\begin{aligned}
\Gamma & \left(\frac{x}{2}+i \frac{\omega}{2 \Omega}\right) \Gamma\left(\frac{x}{2}-i \frac{\omega}{2 \Omega}\right) \\
& =\left|\Gamma\left(\frac{x}{2}+i \frac{\omega}{2 \Omega}\right)\right|^{2} \approx 2 \pi\left(\frac{\omega}{2 \Omega}\right)^{x-1} e^{-\pi \omega / 2 \Omega},
\end{aligned}
$$

thus showing that at high frequencies the spectrum decays exponentially in the special case of $x=1$, and as a power times exponential otherwise. For the particular case of the excited state of $\mathrm{HBr}$, this high frequency approximation turns out to work remarkably well in representing the whole spectrum, as shown by the long-dashed line in Fig. 2.

For the particular case of a single exponential potential and induced dipole, and $x=1$, the asymptotic behavior of the spectra including the effects of thermal averaging has been discussed in Ref. 12, showing that the thermal effects make the spectra fall off more slowly than exponentially. In our case, since we are interested in fixed-energy collisions, no Boltzmann averaging has been performed. This could be, in principle, done formally, but the resulting integrals cannot be expressed by a simple analytic function, except in the high frequency limit, as reported in Ref. 12.

\section{A. Simplification of spectral function}

The importance of the results obtained above is that it provides an analytical expression for the spectrum that makes clear its dependence on the parameters of the molecular system and the collision. The analytical spectral function (8) that we have obtained for a single exponential potential and induced dipole is, however, a bit complicated and not very convenient to use. Hence, in this subsection, we attempt to represent Eq. (7) with an approximate but simpler function,

$$
f_{\mathrm{LB}}(t)=C /\left(1+\frac{t^{2}}{a^{2}}\right)^{s}
$$

which, as we will see, can fit very well the actual behavior of the induced dipole as a function of time during the collision. This particular function $f_{\mathrm{LB}}(t)$ has the same functional form as the velocity autocorrelation in the context of the LevineBirnbaum model, ${ }^{15}$ and accordingly, from now on it will be referred to as the "LB function." " Moreover, previously" it was shown that a LB type function, Eq. (11), was able to give a very good representation of the dipole versus time for collisions between rare gas atoms. Also notice that the LB function clearly has the same functional form as the short time expansion given by Eq. (9).

In order to obtain the value of the parameters of Eq. (11) that gives an accurate representation of Eq. (8), we have several different procedures available. The most accurate one is obviously to perform a numerical least squares fitting of Eq. (7). However, in order to get analytical (but approximate) expressions, we will also introduce a different method based on matching the second and fourth spectral moments. Noting that the $n$th moments of a function $F(\omega)$ are defined by $M_{n}=\int_{-\infty}^{\infty} \omega^{n} F(\omega) d \omega$, where $F(\omega)$ is the Fourier transform of $f(t)$, the following general relation may be derived: ${ }^{16}$

$$
\left.\frac{d^{n} f(t)}{d t^{n}}\right|_{t=0}=\frac{i^{n}}{2 \pi} \int_{-\infty}^{+\infty} \omega^{n} F(\omega) d \omega=i^{n} M_{n} .
$$

Since the above moments cannot readily be evaluated using Eq. (8), we can instead, in view of Eq. (12), use the derivatives $d^{n} f(t) / d t^{n}$ where $f(t)$ is $\mu(r(t))$, given by Eq. (7), evaluated at $t=0$. Thus, we obtain the moments $\hat{M}_{2}$ and $\hat{M}_{4}$, where the hat over the moments mean that they were divided by the 
zeroth-moment $\mathrm{M}_{0}=f(0)=C$,

$$
\hat{M}_{2}=2 E \kappa^{\prime} \kappa / m,
$$

$$
\hat{M}_{4}=4 E^{2} \kappa^{\prime} \kappa^{2}\left(3 \kappa^{\prime}+2 \kappa\right) / m^{2} .
$$

The moments $\hat{M}_{2}$ and $\hat{M}_{4}$ of a single LB function, Eq. (11), are related to the parameters $a$ and $s$ by the following equations: ${ }^{1,15}$

$$
\begin{gathered}
s=\frac{-3 \hat{M}_{2}^{2}}{3 \hat{M}_{2}^{2}-\hat{M}_{4}}, \\
a^{2}=\frac{-6 \hat{M}_{2}}{3 \hat{M}_{2}^{2}-\hat{M}_{4}}=\frac{2 s}{\hat{M}_{2}} .
\end{gathered}
$$

Therefore, by inserting Eqs. (13) and (14) in Eqs. (15) and (16), we find that

$$
\begin{gathered}
s=\frac{3}{2} \frac{\kappa^{\prime}}{\kappa}, \\
a^{2}=\frac{3 m}{2 E \kappa^{2}}=\frac{3}{\Omega^{2}} .
\end{gathered}
$$

The above relations connect the parameters in the single LB model, Eq. (11), with the relevant kinetic parameters of the colliding system, such as the energy $E$ and the range of the potential $\kappa$ and of the induced dipole $\kappa^{\prime}$.

The advantage of using the LB shape is that the spectral function then becomes ${ }^{15}$

$$
F(\omega)=C a \frac{\sqrt{2 \pi}}{2^{s-1}} \frac{(a \omega)^{s-1 / 2}}{\Gamma(s)} \mathrm{K}_{s-1 / 2}(a \omega),
$$

where $\mathrm{K}_{n}(y)$ is the modified Bessel function of the second kind. Equation (19) is clearly more convenient to use than the exact expression, Eq. (8).

In order to illustrate this procedure, we will focus on a particular example corresponding to $\mathrm{H}$ and $\mathrm{Br}$ interacting with a purely repulsive potential function in an $\mathrm{A}^{1} \mathrm{II}_{1}$ excited electronic state. Since the original $a b$ initio data were not available, we roughly estimated the potential as a function of $r$ by fitting a curve in Fig. 1 of Ref. 17 marked $\mathrm{A}^{1} \mathrm{II}_{1}$ to a single exponential potential Eq. (4). Similarly, we scanned a curve in Fig. 3 of Ref. 17 to obtain the dipole function for the same excited state as the potential function, and we fitted to the exponential dipole function Eq. (6). The parameters obtained in the fit are listed in the caption of Table I. Note that for the diatomic molecules studied here the magnitude of the dipole is of the order of $1 \mathrm{D}$ instead of the much smaller overlap dipole of about $0.01 \mathrm{D}$ encountered in collisions of dissimilar rare gas atoms. ${ }^{3}$

In Fig. 1, we get an indication of how well the dipole as a function of time based on the above method can be reproduced by the dipole function obtained from the LSE fit of Eq. (11). This figure shows a dotted curve, Eq. (7), which is the $a b$ initio model. The $a b$ initio model is the ab initio dipole versus separation distance combined with the classical kinetics. The solid curve represents the best LSE fit of the single LB model, Eq. (11), to the ab initio model. The short dashed curve shows the LB model with its parameters determined by matching the moments from the ab initio model using Eqs. (15) and (16). The long dashed curve in the figure is derived from Eq. (9), the short time cosh approximation. We see that the LSE time dependent dipole is somewhat superior overall in its fit to the ab initio model.

Table I compares the values of the parameters obtained in the LSE fitting procedure and the values of the same parameters obtained in the calculation from the moments (see Eqs. (15) and (16)) and the short time approximation for the cosh, Eq. (9). The discrepancies between the parameters for the two curves derived analytically and the curve for the LSE fit are disappointing, despite the fact that all curves lie practically on top of each other. This seems to indicate that there are more than one set of parameters that provide an accurate representation of the time dependent dipole, and suggest that these parameters have a more mathematical than physical meaning. Nevertheless, the spectra obtained from the dipole functions in Fig. 1, whose spectra are shown in Fig. 2, are quite similar and should be useful in identifying CIE spectra in experimental studies. It should be noted that whereas in Ref. 1 the moments of spectral function $|F(\omega)|^{2}$ were calculated from the right side of Eq. (12), to obtain results here in closed form the moments of $F(\omega)$ were calculated from the left side of Eq. (12).

\section{CIE FROM REACTIVE COLLISIONS}

Let us now turn to more realistic situations where neither the interaction potential between the colliding molecules nor the induced dipole can be represented by a single exponential function. In particular, we will focus on the following on collisions between reactive molecules, where the potential is characterized by the presence of a very deep well, deep in the sense that its depth is much larger than the standard thermal energy at room temperature. In order to model this situation, we will focus on an approximated case were the interaction potential can be modeled by a Morse potential. The induced dipole, in general, will also show a non-monotonic behavior since, as mentioned before, it has to satisfy the requirements of being zero both at zero and at infinite separations. In order to deal with this complicated situation we will first discuss a convenient way to represent this non-monotonic induced dipole by using a fit to a sum of exponentials.

Fits to the ab initio dipole moment have been published using exponential, Padé, and polynomial functions. Some results of various approaches to fitting the dipole function are compared in Refs. 9 and 10. In this work, we have chosen to fit the $a b$ initio dipole with the following sum of exponentials:

$$
\mu(r)=\sum_{i=1}^{N} A_{i} e^{-\kappa_{i}^{\prime}\left(r-r_{0}\right)},
$$

where $r$ is the inter-nuclear separation, $r_{0}$ is the equilibrium separation, and $A_{i}$ and $\kappa_{i}^{\prime}$ are the fitting parameters, which for the case of a single exponential dipole would represent the amplitude and the range, respectively. We have chosen this exponential representation for convenience and we find it gives a very accurate fit to the values of the dipole as a function of 


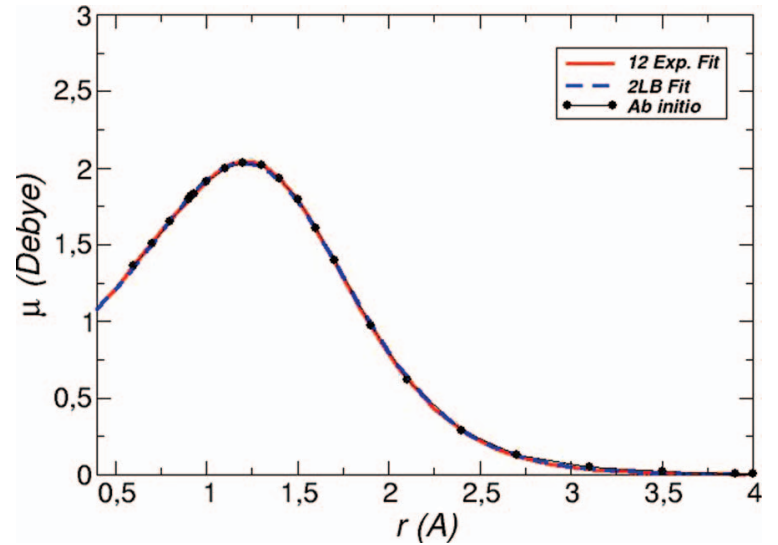

FIG. 3. Dipole versus distance for the ground electronic state of HF. (a) The solid line is the LSE fit of Eqs. (20) and (21) to the ab initio dipole versus separation distance. (b) The dashed line is the LSE fit using the simplified expression (27). The dots mark the ab initio dipole values for HF, taken from Ref. 20.

the inter-nuclear separation. The particular advantage of using this multi-exponential representation is that we can make use of the results derived in Paper I and obtain analytical expression for the spectrum.

In order to reduce the number of fitting parameters it is convenient to replace $\kappa_{i}^{\prime}$ in Eq. (20) by the following form:

$$
\kappa_{i}^{\prime}=b+d(i-1) .
$$

This simple assumption facilitates making the LSE fit stable in converging to a minimum value. We have performed a LSE fit of Eqs. (20) and (21) on the tabulated $a b$ initio data for $\mathrm{HF}^{20}$ and $\mathrm{HBr} .{ }^{17}$ There are some variations among the $a b$ initio dipole values reported by different investigators which depend mainly on the atomic bases functions used in their calculation. Despite these variations among the different approaches, they produced a dipole as a function of time with a single peak. For our representation of the dipole function, we selected the $a b$ initio results covering the greatest range and with the most closely spaced r-values.

It was found that a very accurate fit to the $a b$ initio values of the dipole moments examined here, namely, the halides and $\mathrm{CO}$ and $\mathrm{OH}$, was obtained with 12 exponentials, i.e., $i=1-12$, although in some cases more or less dipole terms sufficed. In order to make the LSE fit more accurate, it was useful to interpolate the ab initio dipole data in ranges where the data were sparse. For some of the higher collision energies, it was necessary to linearly extrapolate the ab initio values of the dipole at the smallest separation distances to obtain a value at the distance of closest approach. Twelve values of $A_{i}$ and values of the two parameters $b$ and $d$ were obtained for each atomic pair. Figure 3 shows the quality of the LSE fit for HF. Values of $b$ and $d$ for the ground electronic state of HF and $\mathrm{HBr}$ atomic pairs are given in Table II.

Using this accurate representation of the dipole function, it is relatively straightforward to evaluate the CIE. As we did in Paper $\mathrm{I}^{1}$ we assume that the atoms in polar diatomic molecules interact via a Morse potential in their ground electronic state,

$$
V(r)=-D+D\left(1-e^{-\kappa\left(r-r_{0}\right)}\right)^{2}
$$

TABLE II. Values of the dipole moment parameters in Eqs. (20) and (21) for the ground electronic states of $\mathrm{HF}$ and $\mathrm{HBr}$. For HF, the fit was calculated for the range $0.0-6.0 \AA$ and for $\mathrm{HBr}$ the fit was calculated for the range 1.0-5.0 A. Neither of these fits are unique, but they are very good representations of the dipole data (in D) and are given here for illustrative purposes. A simple interpolation procedure was used in both cases to generate several thousand data points for the LSE fitting procedure. The sum of coefficients, $\sum_{i=1}^{12} A_{i}$, equals the dipole value at the equilibrium distance $r_{0}$ (see Table III).

\begin{tabular}{llc}
\hline \hline & \multicolumn{1}{c}{$\mathrm{HF}$} & $\mathrm{HBr}$ \\
\hline$b$ & +4.3997 & +1.3157 \\
$d$ & +0.14246 & +1.0991 \\
$\mathrm{~A}_{1}$ & $+5.2177 \times 10^{+7}$ & +0.9452 \\
$\mathrm{~A}_{2}$ & $-4.3467 \times 10^{+8}$ & -18.707 \\
$\mathrm{~A}_{3}$ & $+1.5637 \times 10^{+9}$ & +313.00 \\
$\mathrm{~A}_{4}$ & $-3.1377 \times 10^{+9}$ & -1619.7 \\
$\mathrm{~A}_{5}$ & $+3.7443 \times 10^{+9}$ & +4512.4 \\
$\mathrm{~A}_{6}$ & $-2.5245 \times 10^{+9}$ & -7961.2 \\
$\mathrm{~A}_{7}$ & $+6.4070 \times 10^{+8}$ & +9458.6 \\
$\mathrm{~A}_{8}$ & $+2.9631 \times 10^{+8}$ & -7703.8 \\
$\mathrm{~A}_{9}$ & $-2.3555 \times 10^{+8}$ & +4252.2 \\
$\mathrm{~A}_{10}$ & $+1.4510 \times 10^{+7}$ & -1522.0 \\
$\mathrm{~A}_{11}$ & $+2.7621 \times 10^{+7}$ & +318.88 \\
$\mathrm{~A}_{12}$ & $-6.7969 \times 10^{+6}$ & -29.688 \\
\hline \hline
\end{tabular}

where $D$ is the dissociation energy and gives the minimum of $V(r)$ at $r_{0}$, and we consider only head-on collisions. Then we use the closed form of the kinetic solutions ${ }^{18}$ for separation distance versus time, combined with the $a b$ initio dipole versus separation distance to obtain the dipole as a function of time. These solutions depend on the value of $\rho=1+E / D$, where $E$ is the fixed total energy and $D$ is the depth of the potential well. The two different non-bounded solutions are then

$$
r=r_{0}+\frac{1}{\kappa} \ln \left(\frac{1+\omega_{0}^{2}\left(t-t_{0}\right)^{2}}{2}\right) \quad \text { if } \rho=1,
$$

$r=r_{0}+\frac{1}{\kappa} \ln \left(\frac{\sqrt{\rho} \cosh \left(\gamma\left(t-t_{0}\right)\right)-1}{\rho-1}\right) \quad$ if $\rho>1$,

where $\gamma^{2}=\omega_{0}^{2}(\rho-1), \omega_{0}=\kappa \sqrt{2 D / m}$ and $t_{0}$ is the time at the turning point which for simplicity we will take as our time origin, i.e., $t_{0}=0$. In solving the kinetic problem it was assumed that the effect of the radiation on the trajectory of the accelerating atoms was negligible, a good assumption which is discussed in Appendix A.

For chemically reactive systems like HF, the energy associated with the depth of the well is so high (i.e., $D=5.84 \mathrm{eV}$ for HF) that the approximation $\rho \approx 1$ will hold unless dealing with extremely high energetic collisions (at room temperature, $k T / D \approx 1 / 225$ ). In Appendix $\mathrm{B}$ we will discuss what can be done for highly energetic collisions, for which $\rho>1$.

By substituting the trajectory for $\rho=1$, Eq. (23), into the multi-exponential representation of the dipole, Eq. (20), we obtain the following time-dependence of the induced dipole:

$$
\mu(r(t))=\sum_{i=1}^{N} A_{i}\left(\frac{1+\omega_{0}^{2} t^{2}}{2}\right)^{-x_{i}},
$$




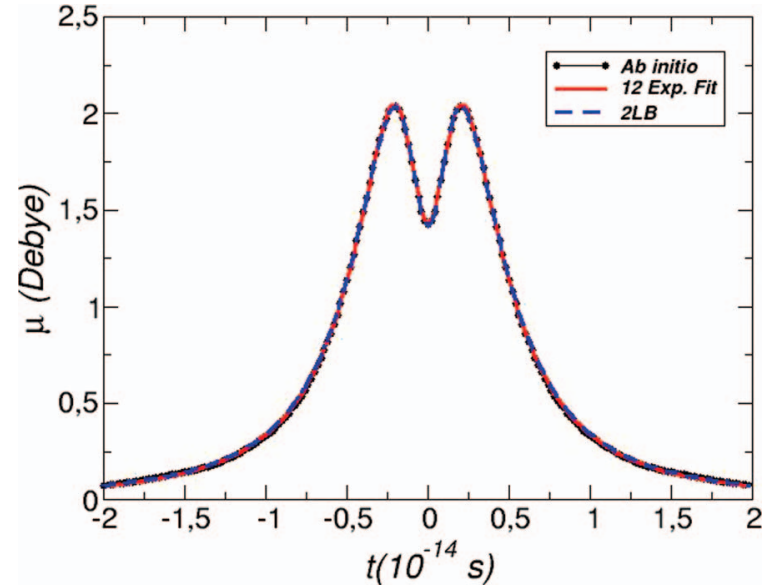

FIG. 4. For the ground electronic state of HF, this figure compares ab initio dipole versus time (dots) with the sum of exponentials fit of the dipole (solid line), Eq. (25), with $\rho=1$, and with the two LB function using the parameters given for Eq. (28) in Table IV. Note that at $t=0$, there is a minimum and at $t= \pm t_{\max }$, there is a maximum.

where $x_{i}=\kappa_{i}^{\prime} / \kappa$. Note the similarity of the terms of this equation to the single LB model, Eq. (11). Figure 4 compares for HF $(\rho=1)$ the ab initio dipole with the sum of exponential (LSE) fitted dipole equation (20) as functions of time. It should not be surprising that the two are virtually indistinguishable because the LSE fit to the $a b$ initio dipole is very good as seen in Fig. 3. The values of the dipole parameters in Eq. (20) are given in Tables II and III. Tables I-III contain values of $\mathrm{HBr}$ parameters in the ground electronic state for comparison with those of HF.

A spectral function $F(\omega)$ is obtained by taking the cosine transform of Eq. (25), ${ }^{13}$

$$
F(\omega)=\sum_{i=1}^{N} \frac{2 A_{i}}{\omega_{0}} \frac{\sqrt{2 \pi}}{\Gamma\left(x_{i}\right)}\left(\frac{\omega}{\omega_{0}}\right)^{x_{i}-1 / 2} \mathrm{~K}_{x_{i}-1 / 2}\left(\frac{\omega}{\omega_{0}}\right),
$$

which is plotted in Fig. 5. The negative portion of this spectrum arises from the declining dipole at very small values of $r$, as seen in Fig. 3. Since this occurs at very short times, its contribution to the spectral function occurs at relatively high frequencies. According to Larmor's theorem the frequency spectrum is proportional to $\omega^{4}|F(\omega)|^{2}$ (see Eq. (1)) which is shown in Fig. 6 for HF $(\rho=1)$. The outstanding feature of this CIE spectrum is the appearance of two peaks in contrast with the single peak spectrum ${ }^{1,4,6}$ produced by the single exponential dipole function that characterizes collisions of dissimilar rare gas atoms.

It is worth emphasizing that the appearance of two peaks in the CIE spectrum is neither an artifact of the fitting pro-

TABLE III. HF and $\mathrm{HBr}$ parameters in the Morse potential, Eq. (22), obtained from Refs. 10, 20, and 21.Values for $\omega_{0}$, calculated from Eq. (9) in Ref. 1, in the ground electronic states. $r_{\min }$ indicates the separation at the turning point of the trajectory corresponding to $\rho=1$.

\begin{tabular}{lcllclc}
\hline \hline & $D(\mathrm{eV})$ & $r_{0}(\AA)$ & $\kappa\left(\AA^{-1}\right)$ & $\omega_{0}\left(10^{14} \mathrm{~s}^{-1}\right)$ & $r_{\min }(\AA)$ & Mass (amu) \\
\hline $\mathrm{HF}$ & 5.84 & 0.9141 & 2.5684 & 8.8463 & 0.6443 & $(20 / 19)$ \\
$\mathrm{HBr}$ & 3.75 & 1.414 & 1.996 & 5.402 & 1.066 & $(81 / 80)$ \\
\hline \hline
\end{tabular}

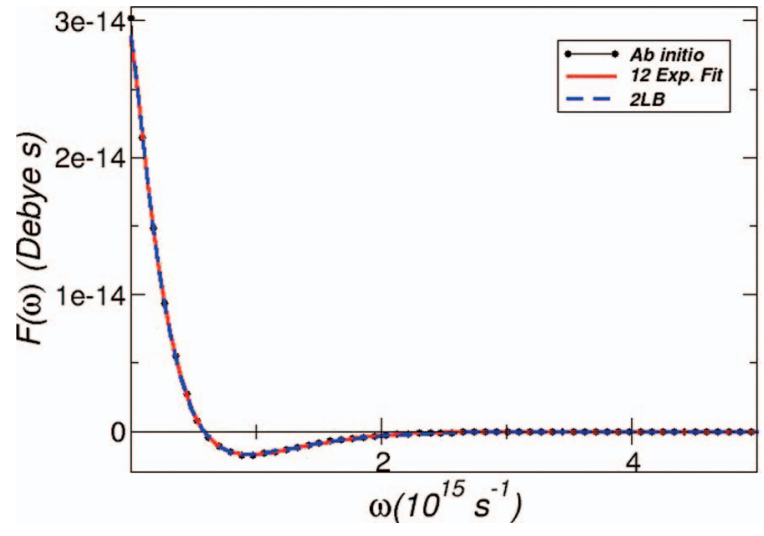

FIG. 5. The cosine transform of Eq. (25), see Eq. (26), of the HF dipole calculated from the sum of exponentials in Fig. 4 (solid line), from the two LB function, Eq. (28) (dashed line), and numerically from the $a b$ initio data the induced dipoles (dots).

cedure nor of the particular choice of the exponential representation used. We have verified this by using a fully numerical procedure in which the $a b$ initio values of the induced dipole were interpolated and the resulting function Fouriertransformed, obtaining the same result. Another important remark is that some simple approximations to the induced dipole, like that of using the derivative of the potential, ${ }^{7}$ are not accurate enough at short distances and cannot capture the existence of these multiple peaks.

The physical origin of this second peak is probably rooted on the change of slope of the induced dipole at short distances, as a reflection of the change in the nature of the HF bond from covalent to ionic. This different slope probably introduces a second characteristic time scale in the problem that eventually leads to the second peak observed in the spectrum. Further investigations to confirm the physical origin of this interesting finding are currently undergoing.

\section{A. Simplified expression for the CIE spectrum}

Despite the successful multi-exponential fit of the ab initio dipole, $\mu(r)$, and the resulting analytical evaluation

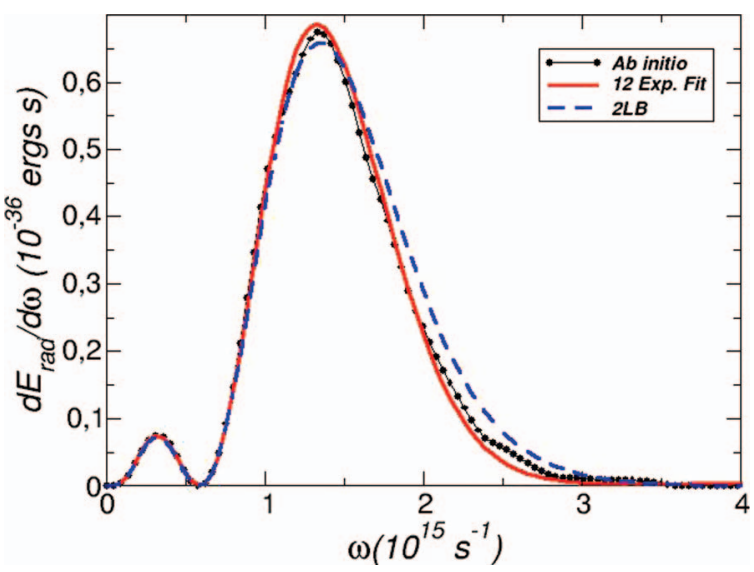

FIG. 6. The CIE spectrum for the ground electronic state of HF with $\rho$ $=1$, obtained numerically from the $a b$ initio data (dots), from the multiexponential fit (solid line), see Eqs. (1) and (26), and from the two LB function, Eq. (30) (dashed line). 
of its spectrum for $\rho=1$, we wished to obtain a simpler expression whose parameters could be related to the kinetic parameters of the collision for any value of $\rho$. We find that it is possible to obtain an excellent fit of the ab initio dipole versus distance, $\mu(r)$, using the following function:

$$
\mu(r)=\frac{C_{1}}{\left(B_{1}+e^{\kappa\left(r-r_{0}\right)}\right)^{s_{1}}}-\frac{C_{2}}{\left(B_{2}+e^{\kappa\left(r-r_{0}\right)}\right)^{s_{2}}},
$$

which involves the 6 fitting parameters: $C_{1}, C_{2}, B_{1}, B_{2}, s_{1}$, and $s_{2}$. The parameters $\kappa$ and $r_{0}$ are taken from the kinetics. This particular functional form has the advantage that using the trajectory for $\rho=1$, the dependence of the induced dipole on time reduces to two LB functions,

$$
\mu(t)=\frac{c_{1}}{\left(1+\left(\frac{t}{a_{1}}\right)^{2}\right)^{s_{1}}}-\frac{c_{2}}{\left(1+\left(\frac{t}{a_{2}}\right)^{2}\right)^{s_{2}}},
$$

where

$$
c_{i}=C_{i}\left(\frac{2 B_{i}+1}{4}\right)^{-s_{i} / 2} \text { and } a_{i}^{2}=\frac{1}{\omega_{0}^{2}}\left(2 B_{i}+1\right) .
$$

Accordingly, by taking the cosine transform of Eq. (28), we get an analytical expression for the spectrum,

$$
F(\omega)=\sum_{i=1}^{2} c_{i} a_{i} \frac{\sqrt{2 \pi}}{2^{s_{i}-1}} \frac{\left(a_{i} \omega\right)^{s_{i}-1 / 2}}{\Gamma\left(s_{i}\right)} \mathrm{K}_{s_{i}-1 / 2}\left(a_{i} \omega\right),
$$

which is now given by the sum of just two functions rather than requiring the $N$ functions of Eq. (26) that arise in fitting the exponential functions, Eqs. (20) and (21), to the ab initio data. For the particular case of HF, the values of the parameters in Eq. (27) obtained by fitting the $a b$ initio data for the induced dipole vs. distance are listed in Table IV. Using these parameters, Figs. 3-6 show how accurately this simplified description matches the induced dipole (Fig. 3), its time dependence (Fig. 4), the spectral function (Fig. 5), and the emission (Fig. 6) of HF. Therefore, by a mere fit to the $a b$ initio data for the induced dipole of a reactive pair of atoms it is possible, using the procedure sketched here, to obtain a simple analytical expression for its CIE spectrum. The new feature of this spectrum for reactive systems is that, in general, and in contrast to standard CIE of rare gases, it will show the presence of more than one peak. This has been verified not only for $\mathrm{HF}$ but also for other halides (HBr, HCL, HI) as well as $\mathrm{OH}$ and $\mathrm{CO}$.

Another interesting finding concerns the particular shape of the spectral function. Previously, ${ }^{1}$ it was shown that a LB type function, Eq. (11), is able to give a very good representation of the dipole versus time for collisions between rare gas atoms. For the collision of chemically reactive atoms, we have also found that the difference of two LB functions gives

TABLE IV. The HF parameter values for the two LB model Eq. (28) for various values of $\rho$.

\begin{tabular}{lcccccc}
\hline \hline$\rho$ & $c_{1}(\mathrm{D})$ & $\mathrm{a}_{1}\left(10^{+14} \mathrm{~s}\right)$ & $s_{1}$ & $c_{2}(\mathrm{D})$ & $\mathrm{a}_{2}\left(10^{+14} \mathrm{~s}\right)$ & $\mathrm{s}_{2}$ \\
\hline 1.0 & 20.705 & 3.6402 & 1.2114 & 15.459 & 2.6093 & 1.7405 \\
1.5 & 7.1054 & 4.7382 & 3.8153 & 5.7343 & 3.2273 & 3.4841 \\
3.0 & 12.899 & 2.7632 & 4.3766 & 11.639 & 94.323 & 6347.1 \\
5.0 & 20.151 & 2.2633 & 5.1417 & 18.971 & 197.01 & 44539 \\
\hline \hline
\end{tabular}

a good representation of the dipole versus time. One of the LB functions has a positive sign and the other has a negative sign. The positive LB in the sum is "slower" than the negative LB function. Roughly speaking, the fast LB is responsible for the dipole increasing with increasing time in the early portion of the half-collision (i.e., at small atomic separation distances) and the slow LB is responsible for the dipole decreasing with increasing time in the later portion of the half-collision (i.e., at large atomic separation distances). It was suggested that the dipole at large separations is due to overlap interactions, whereas the dipole at small separations is due to ionic interactions. . $^{6}$

It may be noted that the transfer of spectral shape developed here is not limited to the use of only LB functions. In fact, any function that fits the induced dipole vs. time data and has suitable mathematical and physical properties may be employed. A good example of such a function is the so called BC shape, see Ref. 19.

\section{DISCUSSION}

In this work, we have extended the results of Paper I to analyze the CIE spectra arising from half collisions in excited electronic states and from reactive collisions. We have shown that accurate analytical expressions for the CIE can be obtained, and provide a useful qualitative insight on its dependence on the relevant physical parameters of the collision. The primary approximations used are the neglect of nonhead-on collisions and quantum effects, and their effect in the spectrum is discussed in Paper I.

We have investigated CIE spectra from collisions of atoms which can chemically bond on impact. According to Kornweitz et al. ${ }^{7}$ the dipole function for HF, for example, is due to a purely ionic bond at short distances. This dipole decreases at larger distances as the binding switches from an ionic to covalent form. This change in the character of the bonding may be regarded as a type of chemical reaction. We have shown that CIE might shed some light on the time behavior of such reactions. The longer time dipole function (i.e., the positive term in Eq. (28)) is similar to that studied in mixtures of dissimilar rare gas atoms, whereas the shorter time portion of the dipole function (i.e., the negative term in Eq. (28)) is new and has not yet received any attention in CIE or CIA. Yet we would expect CIE to appear in photo-dissociation investigations, such as those involving excited electronic states where the potential function is purely repulsive. In this case as emphasized here the CIE spectrum should exhibit a single peak. We have shown that the switch in the slope of the dipole versus separation distance will produce a spectrum with two peaks instead of the single peaked spectrum obtained in collisions of dissimilar rare gas atoms. The dipoles involved in pairs of dissimilar atoms which can chemically bond are large, about the order of a Debye. This and the much higher frequencies involved in CIE from these systems should make such spectra easier to detect than those generated by dissimilar rare gas atoms. Where there is a significant variation in the relative energy of the colliding particles, it may be necessary to integrate the spectrum over this energy distribution. Such an integration is unlikely to lead to an analytic 
expression for the resulting spectrum. Moreover, since the number of radiating partners in one cc gas sample might be many orders of magnitude greater than the concentration attainable in an atomic beam type experiment, it may be possible to observe CIE more readily for the reactively colliding atoms studied in photo-dissociation experiments.

Note added in proof: The following typographic errors were found in Ref. 1: On page 184304-5, Eq. (27), a minus sign should be inserted in front of the exponent $\kappa / \kappa^{\prime}$. On page 184304-3, Eq. (15), in the denominator +1 should be replaced by -1 . On page 184394-7, in the column of text on the right hand side of the page, all "v" should be replaced by " $v . "$

\section{ACKNOWLEDGMENTS}

It is a pleasure to thank Professor R. D. Levine for his suggestions particularly for the use of half collisions to study CIE in diatomic molecules, Professors W. M. Gelbart, C. M. Knobler, and especially H. Reiss whose support made this work possible, and Dr. Aron Yoffe for assistance with some of the calculations. This work has been supported by the MICINN of the Spanish government through the I3 Program and Grants Nos. FIS2008-01299 and FIS2011-22603 co-financed by European Union's FEDER funds.

\section{APPENDIX A: NEGLIGIBLE EFFECT OF RADIATION ON TRAJECTORY}

Following Jackson, ${ }^{8}$ (Sec. 17.1) we consider when the effect of radiation on the trajectory from accelerating dipoles in a pair of atoms becomes important. The energy imparted by the forces acting between the particles is of the order,

$$
E_{k i n} \sim m(a T)^{2},
$$

and from Larmor's theorem the energy radiated $E_{\text {rad }}$ due to the acceleration of a charge $e$ during an interval of time $T$ is

$$
E_{\text {rad }} \sim \frac{2\left(\mu \kappa^{\prime}\right)^{2} a^{2} T}{3 c^{3}},
$$

where $e$ has been replaced ${ }^{3}$ here by $\mu \kappa^{\prime}$. The interval of time $T$ is taken as the duration of collision, $1 / \kappa v$, where $v$ is the relative velocity. Radiative effects become important when $E_{\text {rad }}$ $\gtrsim E_{k i n}$. Then Eqs. (A1) and (A2) give

$$
T \lesssim \tau=\frac{2}{3} \frac{\left(\mu \kappa^{\prime}\right)^{2}}{m c^{3}},
$$

where $\tau$ is a characteristic time of the system.

For $\mathrm{HBr}^{10,20}$ with $\mu=0.937 \mathrm{D}, \kappa^{\prime}=5.35 \times 10^{8} \mathrm{~cm}^{-1}$, and $m=1.65 \times 10^{-24} \mathrm{gr}$, we find that $\tau=3.48 \times 10^{-25} \mathrm{~s}$, whereas the time $T=2.20 \times 10^{-15} \mathrm{~s}$, the collision duration, is so much longer than $\tau$ that the effect of CIE radiation on the trajectory should not be important.

\section{APPENDIX B: HIGH ENERGY COLLISIONS IN REACTIVE SYSTEMS}

As mentioned previously, for chemically reactive atoms the value of the well depth, $D$, is so high that $\rho=1+(E / D)$ $\approx 1$ is a useful approximation in practically all cases. However, there has recently been a lot of interest in the emission from extremely energetic (supersonic) beams. In this section, we will discuss different approximations that can be done to describe analytically the CIE arising from highly energetic collisions in reactive systems.

\section{CIE from the multiexponential fit and high-frequency approximation}

For these high energy collisions with $\rho>1$, using the corresponding solution for the trajectory, Eq. (24), and substituting into the multi-exponential function, Eqs. (20) and (21), we obtain for the dipole as a function of time,

$$
\mu(r(t))=\sum_{i=1}^{N} A_{i}\left(\frac{\rho-1}{\sqrt{\rho} \cosh (\gamma t)-1}\right)^{x_{i}},
$$

where $\gamma^{2}=\omega_{0}^{2}(\rho-1)$. A particular example of the timedependent dipole corresponding to $\rho=3$ is shown in Fig. 7 for HF.

Unfortunately, Eq. (B1) cannot be Fourier transformed analytically, so a different strategy must be used to gain some useful information in this case. A straightforward option is to get information about the high frequency behavior by using a short time approximation. After substituting the short time expansion for the cosh $(\gamma t)$ term in Eq. (B1), we obtain a result that is similar in form to Eq. (9),

$$
\mu(r(t))=\sum_{i=1}^{N} A_{i}\left(\frac{\sqrt{\rho}+1}{1+\gamma^{2} t^{2} /(2-2 / \sqrt{\rho})}\right)^{x_{i}} .
$$

This expression is plotted in Fig. 7 for the particular case of HF for $\rho=3$. One can see in the figure that, in clear consistency with the approximation involved, the dipole is only accurately represented at very short times. The cosine transform of Eq. (B2) is then in the functional form of the approximation given in Eq. (10). The actual spectrum is obtained by squaring

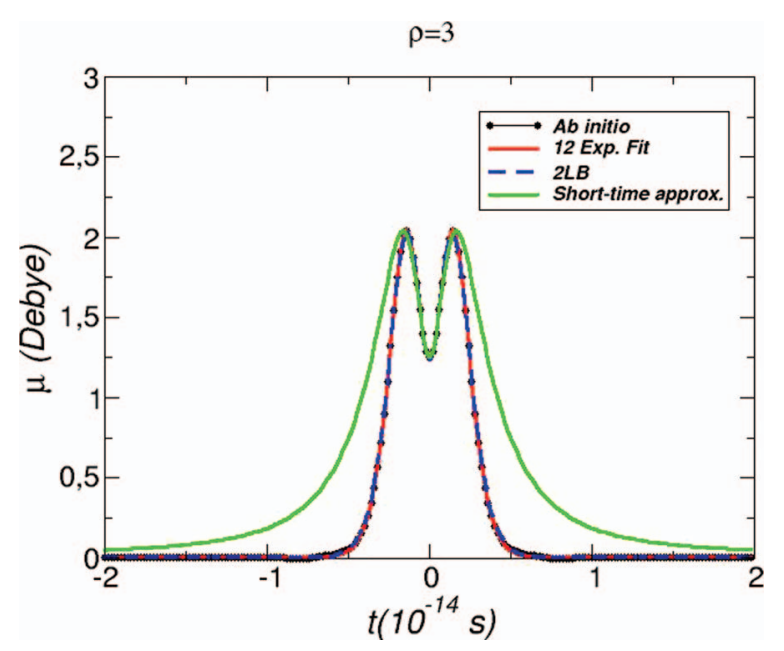

FIG. 7. Dipole versus time for the ground electronic state of HF corresponding to $\rho=3$, obtained numerically from an interpolation of the ab initio data (dots); from the multi-exponential fit (solid line), Eq. (B1); from the simplified two LB dipole model (dashed line), Eq. (B3); and using the shorttime approximation to the sum of exponentials (green line), Eq. (B2). The curve produced by the short-time approximation deviates noticeably from the $a b$ initio values as time increases. Note that at $t=0$, there is a minimum and at $t= \pm t_{\max }$, there is a maximum. 


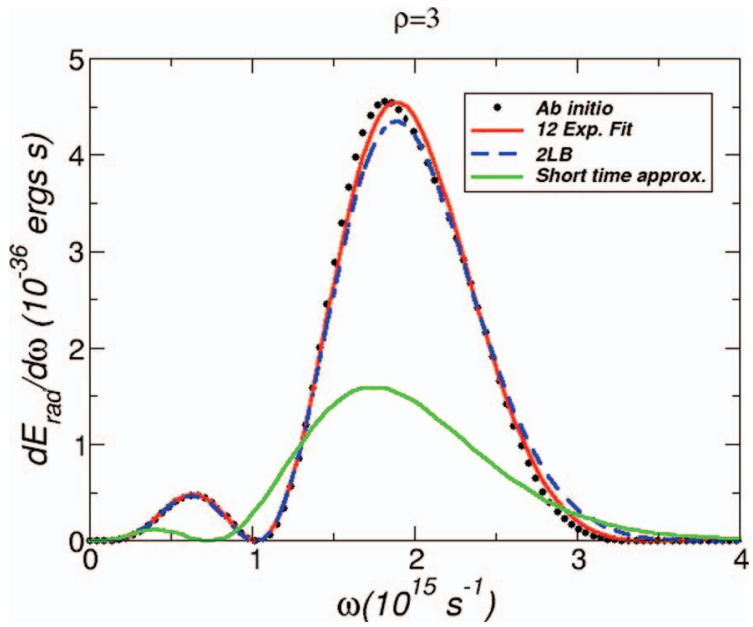

FIG. 8. The CIE spectra for the ground electronic state of HF corresponding to $\rho=3$, obtained numerically from an interpolation of the $a b$ initio data (dots); from the numerical Fourier transform of the multi-exponential fit (solid line), Eq. (B1); from the simplified two LB dipole model (dashed line), Eq. (B5); and using the short-time approximation to the sum of exponentials (green line), Eq. (B2). Compare with Fig. 6.

the transform and multiplying by $\omega^{4}$ as shown in Eq. (1). A plot of this high-frequency approximation spectrum is shown in Fig. 8 for $\mathrm{HF}$ and $\rho=3$, evidencing that this approximation does not provide a good representation of the spectrum.

\section{CIE from the simplified fit}

Instead of the more complicated multi-exponential fit, one can use the simplified two term expression for $\mu(r)$, Eq. (27), and substitute the trajectory $r(t)$, Eq. (24), to obtain a simpler expression for the time-dependent dipole,

$$
\mu(r(t))=\sum_{i=1}^{2} C_{i}\left(\frac{\rho-1}{B_{i}(\rho-1)+\sqrt{\rho} \cosh (\gamma t)-1}\right)^{s_{i}} .
$$

This expression is plotted in Fig. 7 for the particular case of HF for $\rho=3$, but once again it cannot be transformed analytically to get the spectrum.

\section{Simplified expressions for the spectrum}

We have just seen that for the case $\rho>1$, it is not possible to obtain a simple analytical expression for the spectrum using neither a multi-exponential fit nor the simplified representation of the induced dipole versus distance. In our goal to obtain a simpler expression we found that it is possible to obtain an excellent fit of the dipole versus time, $\mu(t)$, in all cases by using a functional representation similar to the two LB model, given by Eq. (28), namely,

$$
\mu_{f i t}(t)=\frac{d_{1}}{\left(1+\left(\frac{t}{b_{1}}\right)^{2}\right)^{n_{1}}}-\frac{d_{2}}{\left(1+\left(\frac{t}{b_{2}}\right)^{2}\right)^{n_{2}}} .
$$

As a consequence, we discovered that the following spectral function:

$$
F(\omega)=\sum_{i=1}^{2} d_{i} b_{i} \frac{\sqrt{2 \pi}}{2^{n_{i}-1}} \frac{\left(b_{i} \omega\right)^{n_{i}-1 / 2}}{\Gamma\left(n_{i}\right)} \mathrm{K}_{n_{i}-1 / 2}\left(b_{i} \omega\right),
$$

which is similar to the $2 \mathrm{LB}$ spectral function given by Eq. (30), is an accurate representation of the spectra for reactive collisions for all values of $\rho$.

To evaluate the parameters $d_{1}, d_{2}, b_{1}, b_{2}, n_{1}$, and $n_{2}$, involved in the functions for the case $\rho>1$, the most straightforward protocol involves the direct LSE fit of the time-dependent dipole for a given value of $\rho>1$, given by Eqs. (B1) or (B3) to Eq. (B4). The dotted curve in Fig. 7 represents the fit of the $a b$ initio data as a function of time using the kinetic solution for $r(t)$. Note the dip in the value of the dipole as the time $t$ approaches zero. In this way, one can get an excellent representation of the spectra, as shown in Fig. 8.

Figure 9, computed using the above protocol, shows how the CIE spectrum of HF, for example, varies with $\rho$. A similar result should be expected for any other molecule whose dipole as a function of $r$ exhibits a peak. The parameter $\gamma$ is a measure of the duration of collision and its inverse is related to the spectral width. Subscript 1 is taken to represent the positive LB term. Regarding the higher frequency spectral feature, we note that with increasing $\rho$, increasing velocity, or increasing kinetic energy, the duration of collision decreases and the spectral width increases. Also, the increase in width is accompanied by an increase in the peak amplitude and peak frequency.

LSE fits obtained in this manner are very good, but are not unique. There are six parameters that need to be determined and the data used in the LSE fit are only enough to determine four uniquely. Two of the parameters, most conveniently $d_{1}$ and $d_{2}$, must be determined by additional relationships to have a unique set. For example, requiring the value of the dipole at the closest separation distance $(t=0)$ and the value of the dipole at the time the dipole reaches its maximum $\left(t=t_{\max }\right)$ to be specified in advance allows the LSE fitting procedure to produce a unique set of the six parameters in Eq. (B4), see Figs. 4 and 7. Not surprisingly, the 2 LB

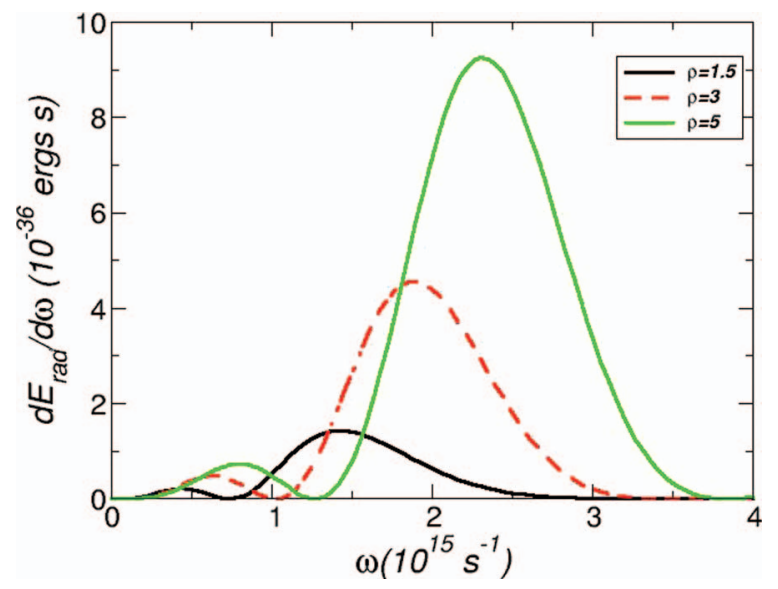

FIG. 9. The CIE spectra for the ground electronic state of HF using the two LB model, Eq. (B5), with $\rho=1.0,1.5,3$, and 5, respectively. Computed from Eqs. (1) and (B5) with the parameters from the LSE fit given in Table IV. 
equivalent of the procedure outlined earlier (see Eqs. (15) and (16)) using the second and fourth moments to calculate the LB parameters does not give very good results when expanded to the sum of two LB functions.

${ }^{1}$ D. Reguera and G. Birnbaum, J. Chem. Phys. 125, 184304 (2006).

${ }^{2}$ J. van Kranendonk, Physica 23, 825 (1957).

${ }^{3}$ L. Frommhold, Collision-Induced Absorption in Gases (Cambridge University Press, 1993), and references therein.

${ }^{4}$ A. Gross and R. D. Levine, J. Phys. Chem. 107, 9567 (2003).

${ }^{5}$ A. Gross, M. Kjellborg, and R. D. Levine, J. Phys. Chem. 108, 8949 (2004).

${ }^{6}$ A. Gross and R. D. Levine, J. Chem. Phys. 119, 4283 (2003).

${ }^{7}$ H. Kornweitz, A. Gross, G. Birnbaum, and R. D. Levine, Phys. Scr. 73, C1 (2006).

${ }^{8}$ J. D. Jackson, Classical Electrodynamics (John Wiley \& Sons, New York, 1963).

${ }^{9}$ G. Herzberg, Molecular Spectra and Molecular Structure I. Spectra of Diatomic Molecules, 2nd ed. (Twelfth printing, Princeton, NJ, 1950), p. 97.
${ }^{10}$ J. F. Ogilvie, The Vibrational and Rotational Spectrometry of Diatomic Molecules (Academic Press, San Diego, 1998), Sec. 7.1.

${ }^{11}$ I. I. Turgov and V. F. Chernyavsky, J. Mol. Spectrosc. 99, 302 (1983).

${ }^{12}$ A. G. Basile, C. G. Gray, B. G. Nickel, and J. D. Poll, Mol. Phys. 66, 961 (1989).

${ }^{13}$ A. Erdelyi, W. Magnus, F. Oberhettinger, and F. G. Triconi, Tables of Integral Transforms (McGraw-Hill, New York, 1954).

${ }^{14}$ Handbook of Mathematical Functions, Applied Mathematical Series Vol. 55, edited by M. Abramowitz and I. A. Stegun (National Bureau of Standards, Washington, DC, 1964).

${ }^{15}$ H. B. Levine and G. Birnbaum, Phys. Rev. 154, 86 (1967).

${ }^{16}$ D. C. Champeney, Fourier Transforms and Their Physical Applications (Academic Press, Great Britain, 1973), Sec. 2.4, p. 16.

${ }^{17}$ D. A. Chapman, K. Balasubramanian, and S. H. Lin, Chem. Phys. 118, 333 (1987).

${ }^{18}$ W. C. DeMarcus, Am. J. Phys. 46, 733 (1978).

${ }^{19}$ G. Birnbaum and E. Cohen, Can. J. Phys. 54, 593 (1976).

${ }^{20}$ J. F. Ogilvie, W. R. Rodwell, and R. H. Tipping, J. Chem. Phys. 73, 5221 (1980).

${ }^{21}$ G. Gaydon, Dissociation Energies, 3rd ed. (Chapman and Hall, London, 1968). 